\title{
Very long-term of survival, 5 years and more in diffuse intrinsic pontine brainstem gliomas in children and adolescents treated with Radiotherapy and Nimotuzumab
}

\begin{abstract}
Diffuse intrinsic brainstem gliomas have a bad prognosis, and short-term survival time. Radiotherapy has been the principal treatment, and chemotherapy has not improved outcome. The anti-EGFR monoclonal antibody Nimotuzumab combined with Radiotherapy was tested in a series of 41 children and adolescents with diffuse intrinsic pontine gliomas (DIPG) included between January 2008 and December 2015 and a follow-up till January 2021. They were irradiated in the Instituto Nacional de Oncologia y Radiobiologia, Havana, Cuba with a median dose of $54 \mathrm{~Gy}$. Nimotuzumab was applied at a dose of $150 \mathrm{mg} / \mathrm{m} 2$, weekly during the period of irradiation, then every 2 weeks by 8 doses, and them monthly for 1,2 or more years. A response was observed in $87.8 \%$ of patients. Prolonged use of Nimotuzumab was feasible and well tolerated. Median age at diagnosis was 7 years old, median survival was 18.8 months. There were minor toxicities, only Grade I or II. Survival rate at 5 years was $34.1 \%$, stablished till years or more. Two relapsing patients were reirradiated. The combination of irradiation and Nimotuzumab is an option to increase survival in DIPG.
\end{abstract}

Keywords: Irradiation of brainstem glioma, Nimotuzumab in brainstem gliomas, very long survival, re-irradiation
Volume 8 Issue 2 - 2021

\author{
Alert J,' Chon I,' Valdes J, Ropero R, ${ }^{2}$ Perez \\ $M,{ }^{4}$ Garcia D, ${ }^{4}$ Forteza M, ${ }^{4}$ Avila ${ }^{3}$ \\ 'Radiotherapy Department, Instituto Nacional Oncologia y \\ Radiobiologia, Havana, Cuba \\ 2J.M. Marquez Hospital, Cuba \\ ${ }^{3}$ Clinical Investigations Department, Instituto Nacional \\ Oncologia y Radiobiologia, Cuba \\ ${ }^{4}$ Oncopediatric Department, Instituto Nacional Nacional \\ Oncologia y Radiobiologia, Cuba
}

\begin{abstract}
Correspondence: Jose Alert, Instituto Nacional Oncologia y Radiobiologia, 17 number I58, apt 8,Vedado, Havana, Cuba,
\end{abstract} Tel 53-78330584,Email jalert@infomed.sld.cu

Received: April 09, 2021 | Published: June 21, 2021

\section{Introduction}

Brainstem tumors in children and adolescents are in most cases diffuse intrinsic pontine gliomas (DIPG), with a defined clinical presentation (multiple bilateral cranial nerve deficits, specially VI and VII, corticospinal tract deficit and ataxia) and characteristic appearance in imaging findings, so histological confirmation are often considered unnecessary. ${ }^{1-9}$ Focal radiotherapy (RT) has been the principal treatment, because surgical resection is not possible, but it has shown a low degree of efficacy, with short-term response and a median survival time of one year or less.In general, 2 and 5-year survival rates from the diagnosis are $10 \%$ and $1 \%$, respectively. ${ }^{1-3,6,10,11}$ DIPG must be distinguished from other subsets of pontine gliomas, such as focal tumors which are described with better prognosis and longer survival. ${ }^{12-15}$ The association of radiotherapy and chemotherapy, in general, has not improved survival. ${ }^{6,16-21}$

We investigate the association of radiotherapy and Nimotuzumab, formerly called h-R3, an humanized monoclonal antibody developed at the Centro de Inmunologia Molecular, in Havana, Cuba and tested the hypothesis that this combination will improved survival. The antibody was obtained by humanization of the murine antibody EGF/ R3.22 Because Nimotuzumab has a 10 fold lower affinity to EGFR as compared to Cetuximab, its capacity to bind EGFR is heavily dictated by cell receptor density. ${ }^{23}$ A distinguishing feature of Nimotuzumab compared to other MAbs of the EGFR class, is the lack of severe skin toxicity and the possibility to be used beyond progression. . $^{1,2425}$ Nimotuzumab preclinical and clinical characterizations has been summarized before. ${ }^{26-29}$ Previous reports with the use of RT and Nimotuzumab show that this association is useful and even improve survival in DIPG. $1,4,6,24,3-34$
Long-term survival with DIPG is defined to occur 2 or 3 years since the time of the initial treatment, and Very Long-term survival is defined to an overall survival (OS) of 5 years or more. ${ }^{2,3,6,8,13,21,35-37}$

\section{Material and methods}

This is a prospective, non-randomized clinical study with a treatment group of 41 children and adolescents (age range between 2 to 18 years old), recruited from January 2008 to December 2015, and a follow-up till January 2021, with the diagnosis of DIPG documented clinically and by imaging (MRI,CT scan). Biopsy and histology confirmation was not a requirement of this study. All patients had a history of cranial nerve paresis, ataxia, lower or upper extremity paresis and characteristics imaging showing tumor primarily located in the pons, and infiltrating diffusely the pons. Others inclusion criteria were Karnofsky-Lansky status $\geq 30 \%$, no pregnancy or breastfeeding. Patients with focal lesions of the brainstem were not elected for the study, they were also excluded those who had received prior chemotherapy or RT. Latter, we decided to include patients, no matter if Karnofsky-Lansky were $20 \%$ or less.

The study was approved by the Ethical Institution Committee at the Instituto Nacional de Oncologia y Radiobiologia (INOR) in Havana, and the Informed Consent obtained from the patients' parents. All patients were irradiated at the INOR and received the monoclonal antibody Nimotuzumab at INOR or at Pediatric Hospital "Juan M. Marquez", also in Havana.

No surgical treatment was feasible; in 3 patients there were pathological results by biopsy; in all patients,tumor was extended to the pons. 
Patients received RT in a lineal accelerator, Gross Tumor Volume (GTV) was defined as the visible tumor, either by MRI or CT, Clinical Target Volume (CTV) accounted for subclinical microscopic disease, generally $1.5 \mathrm{~cm}$ from the GTV and planning Target Volume (PTV) was $0.3-0.5 \mathrm{~cm}$ from GTV, and could vary according to Organ at Risk. RT doses ranged from 54 to $59,8 \mathrm{~Gy}$, with 1,8Gy dose per fraction, 5 doses per week. Six patients were planned with IMRT and the rest with 3D CT, in all patients a thermoplastic mask was fitted. Thirty patients $(73,2 \%)$ received dexamethasone during irradiation time, and radiation dose was recorded weekly.

Nimotuzumab was administered at a dose of $150 \mathrm{mg} / \mathrm{m}^{2}$ (IV) weekly during the time they were receiving RT, beginning in the first day of irradiation, then every 2 weeks per 8 doses, then monthly for one year. In some of the patients, the application of Nimotuzumab was prolonged for 2 o more years, depending of the Institution which made the follow-up. Categorical data were expressed as percent; survival data were estimated using the Kaplan-Meier method with long rank test. The Youden Index was applied to obtain the cut-off point signs of symptomatic improvement dose. Values with $\mathrm{p}<0.05$ was considered statistically significant.

All patients completed the irradiation plan, except 2 who experienced progression of the disease and died with doses of 26 and $34,2 \mathrm{~Gy}$. When there were interruptions in the time of irradiation, the dose of 54 Gy was increased to 57 Gy or more.

A total of 36 patients $(87.8 \%)$, of which 11 patients of the longterm follow- up and 25 of the rest (deceased), received the complete Nimotuzumab schema: interruptions of Nimotuzumab administration were because patients did not concur to the treatment during followup in a timely manner, but those patients received a minimum of $80 \%$ of the doses of the Nimotuzumab.

Two patients were re-irradiated for relapse of the tumor: one at 4 years of the first irradiation with a dose of $54 \mathrm{~Gy}$, the other received 34,2 Gy 3 years after first irradiation; procedures were the same for the first treatment, the bean geometry for re-irradiation was chosen in order to protect previous irradiated areas.

\section{Results}

The median age at diagnosis of the 41 patients included in the series was 7 years old ( $95 \%$ CI, 5-9) with a range between 2 and 18 years old .In all cases tumor was extended to the pons; 22 were male patients (53.7\%) and 19 female patients (46.3.\%).

Karnofsky-Lansky Performance Status was evaluated at inclusion: KLPS $<60, \mathrm{n}=11(26.8 \%), \mathrm{KLPS}=60, \mathrm{n}=14(34.1 \%), \mathrm{KLPS}>60, \mathrm{n}=16$ (39.1\%).

According to RT dose: the range more used was $54-57 \mathrm{~Gy}(\mathrm{n}=17$, 41.5\%); 15 received 57.1-59 Gy (36.6\%) and 9 patients were irradiated with a dose higher than 59 Gy (22.9\%) (Figure 1).

Median dose to first signs of symptomatic improvement was 1,700 cGy, $(95 \% \mathrm{CI}, 1,200-2,200)$ in the group of 14 patients with very long OS. The median dose in the deceased patients was $1,980 \mathrm{cGy}(95 \% \mathrm{CI}$, $1,110-2,550)$. There was no difference with statistical significance $(\mathrm{p}=$ 0.355 ). The point cut-off was established as $1,700 \mathrm{cGy}$. Although univariate analysis for range of improvement dose had not reached difference with statistical significance, it's clinically important to note that for patients that obtained first sign of symptomatic improvement with dose less or equal to 1,700 , the median of time survival was not reached. The survival rates for patients that got improvement with a range dose $\leq 1,700 \mathrm{cGy}$, were $75.0 \%, 66.7 \%$ and $58.3 \%$ versus $75.0 \%$,
$36.7 \%$ and $25.0 \%$ at 1,2 and 5 years, for patients that obtained first signs of symptomatic improvement with range of dose $\geq 1,700 \mathrm{cGy}$, respectively (Figure 2).

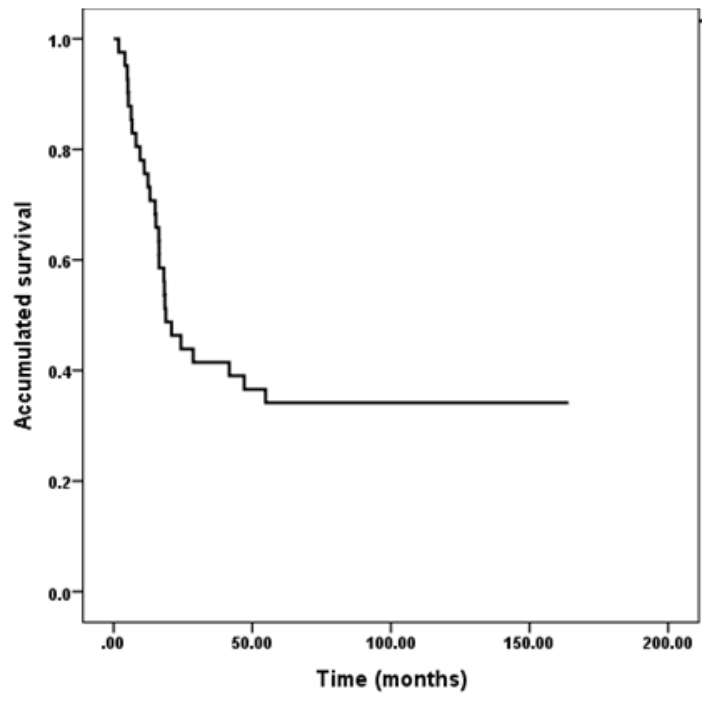

Figure I Overall survival. Diffuse intrinsic pontine brainstem gliomas in children and adolescents treated with Radiotherapy and Nimotuzumab.

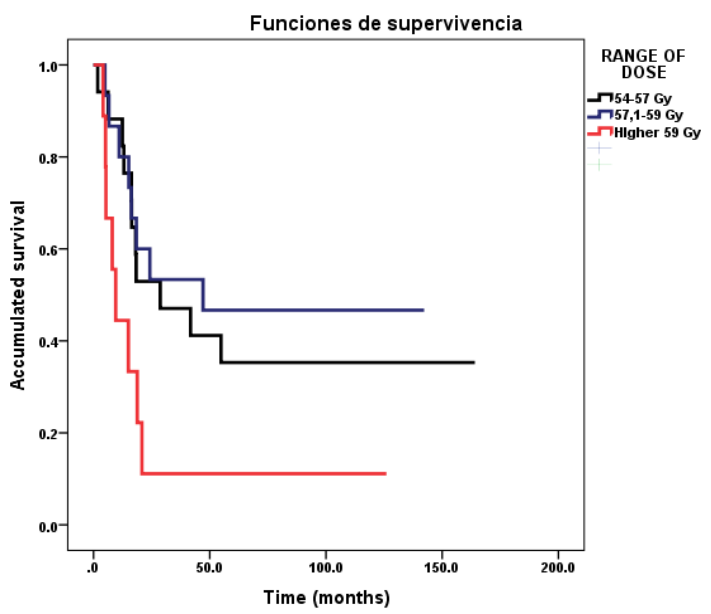

Figure 2 Survival according to range of dose. Diffuse intrinsic pontine brainstem gliomas in children and adolescents treated with Radiotherapy and Nimotuzumab.

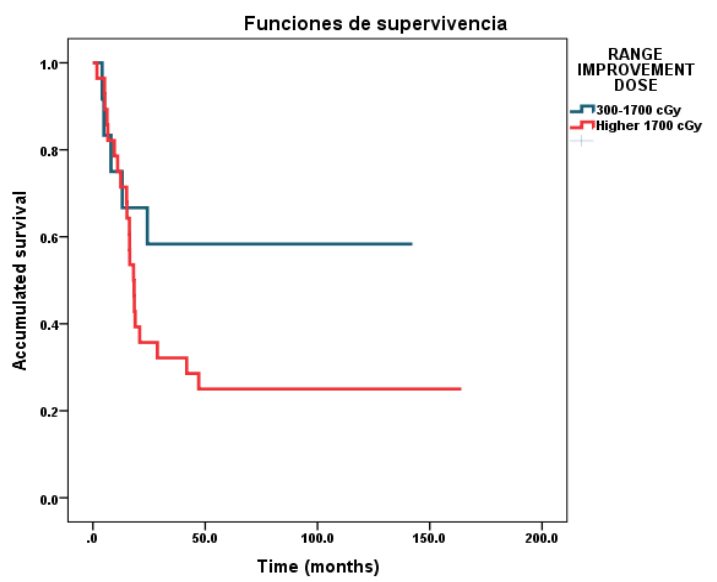

Figure 3 Survival according to range of improvement dose. Diffuse intrinsic pontine brainstem gliomas in children and adolescents treated with Radiotherapy and Nimotuzumab. 
Table I Characteristics and current activities of patients alive with long-term survival

\begin{tabular}{llllllll}
\hline $\begin{array}{l}\text { Age diagnostic } \\
\text { (years) }\end{array}$ & $\begin{array}{l}\text { Current age } \\
\text { (years) }\end{array}$ & Sex & KL & $\begin{array}{l}\text { RT dose } \\
\text { Gy }\end{array}$ & $\begin{array}{l}\text { RT improvement } \\
\text { dose cGy }\end{array}$ & $\begin{array}{l}\text { Time survival } \\
\text { (month) }\end{array}$ & $\begin{array}{l}\text { Current } \\
\text { activity }\end{array}$ \\
\hline 9 & 23 & male & 80 & $54 *$ & 2400 & 164.1 & University \\
5 & 17 & female & 60 & 57.4 & 1600 & 142.2 & -- \\
2 & 14 & female & 70 & 55.8 & 1800 & 141.4 & -- \\
2 & 13 & male & 50 & 57.6 & 1080 & 137.1 & studying \\
7 & 18 & female & 50 & 59.4 & 1440 & 126.0 & studying \\
18 & 28 & female & 50 & 57.4 & 1080 & 122.6 & working \\
5 & 15 & female & 80 & 57.0 & 1440 & 119.4 & studying \\
2 & 12 & female & 30 & 54.0 & 900 & 118.2 & studying \\
13 & 23 & male & 80 & 57.6 & 2700 & 114.9. & University \\
6 & 15 & female & 70 & 57.6 & 1260 & 106.7 & studying \\
10 & 19 & male & 90 & 54.0 & 2200 & 104.5. & studying \\
15 & 23 & male & 50 & 57.4 & 3200 & 99.7 & --- \\
16 & 22 & female & 50 & 54.0 & 3200 & 68.6 & University \\
18 & 23 & male & 80 & 54.0 & 2200 & 61.3. & University \\
& & $*$ Re-irradiation 33.0 Gy & & &
\end{tabular}

Median overall survival from the beginning of treatment was 18.8 months ( $95 \%$ CI, 11.1-25.5); survival rates were $75,6 \%, 41,3 \%$, $36,6 \%$ and $34,1 \%$ at $1,2,3,4$ and 5 years respectively, estimated till 31 January 2021 (Figure 2). At present, 14 patients of the series are alive $(34,1 \%)$. We were able to ascertain vital status of those patients who are alive at a minimum of 5 years, of them, 11 are incorporated to studies, including 4 who are attending studies at University.

Two patients were re-irradiated, one of them survival 9 years after that and is alive and studying at University, and the other improved with the re-irradiation, but died after 8 months.

Treatment was well tolerated and no grade 3 or higher grade toxicity was observed. The most frequent adverse event was alopecia in irradiation fields, observed in 35 patients $(85,3 \%)$. Other reported adverse events were vomiting, head-ache, fever, tremor and nausea in less than $46 \%$ of patients.

There were progression of the tumor in 2 patients, so they not completed the irradiation plan. In the 39 remaining patients there were clinical and imaging response in 36(92\%).

\section{Discussion}

Brainstem gliomas are an heterogeneous group of tumors occurring predominately in children, and generally they are diffuse intrinsic pontine gliomas (DIPG), with a peak between 6 and 7 years of age $(6,8,40)$, primarly located in pons, infiltrating diffusely the pons $(2,6,30,31)$ with a CT and MRI and clinical signs and symptoms that has been considered sufficient for diagnosis (1-9); because of the anatomical location of the tumor biopsy is risky and a surgical resection is not possible, but in the last years there is a tendency to do it $(3,36,37,38)$.

Focal radiotherapy has been considered the standard treatment, ${ }^{1-3,5,8,35,37}$ offering certain benefits, but short-time response, with a bad prognosis and a median survival of one year or less, and few beyond 2 years. . $^{1-3,6,8-10,12,13,32,33,35,37,39} \mathrm{~A}$ radiation dose escalation does not improve outcome and neither chemotherapy nor hypofractionation have improved results. ${ }^{2,6,14,16-18,20,21}$

Nimotuzumab is an anti-EGFR humanized monoclonal antibody that blocks EGF, with anti-proliferative, antiangiogenic and proapoptotic activity, ${ }^{29}$ which explain the results observed in different reports. $1,4,6,7,24,28,30,31-34$ Nimotuzumab has demonstrated that does not produce toxicities, as commonly associated with other EGFR targeting agents, such as skin rash; condition that has allowed to perform combination with other drugs. ${ }^{1,4}$ Adverse events as alopecia, head-ache and nausea could be associated to the irradiation. ${ }^{6,32,33}$ Nimotuzumab combined with RT and continued after the period of irradiation for 1,2 or more years, was well tolerated, its prolonged use is safe and feasible, ${ }^{6,24,32,33}$ can be administered in outpatient settings, ${ }^{6}$ so permitting to attend school, or work. At this moment (Jan 2021), 11 patients of the Very Long Term Follow - up are in different levels of scholarship, of which 4 are attending at the University, only 1 patient of Very long-term follow-up have impairment of knowledge and are receiving special education.

Most patients experienced an improvement in neurological symptoms, it has been reported even in $80 \%{ }^{40}$ In $38(96,7 \%)$ of our patients there were an improvement : in the group of Very Long Overall Survival, it appeared at a median dose of $1,700 \mathrm{cGy}$. In the deceased group the median dose were improvement appeared was 1,980cgy, with not statistical significate difference. It has been suggest that low cumulative doses of RT, can provide neurological improvement. ${ }^{31,40}$

Rate survival results at long-term follow-up $(76,6, \%, 46,3 \%, 41,3 \%, 36,1 \%$ and $34,1 \%)$ at $1,2,3,4$ and 5 years, respectively, established till 10 or more years with the combined treatment of RT and the Nimotuzumab, were never reported previously. in DIPG. ${ }^{39}$ Jackson et al. ${ }^{35}$ reported only $2.6 \%$ at 5 or more years; Hoffman et al. ${ }^{5}$ in a Collaborative Report from the International and European Society for Pediatric Oncology DIPG Registries inform 
about $2,2 \%$ of survival at 5 years; Hassan et al. ${ }^{13}$ reported only $7,5 \%$ at 3 years. Crotty et al., ${ }^{21}$ from the Seattle Children's Hospital reported $\mathrm{O} \%$ of OS at 5 year

It's important to remark that 36 patients $(87,8 \%)$ received the Nimotuzumab doses programmed, with minor interruptions, so the anti-proliferative, anti-angiogenic and pro-apoptotic activity of the product $^{29}$ were present during irradiation period and later during one, two or more years, and probably explain the very long overall survival.

When the disease progress, re-irradiation has been employed, and has been reported to give a certain survival benefit. ${ }^{1,41-43}$ In our 2 patients were re-irradiation was combined with Nimotuzumab, one is alive more than 5 years after the procedure, the other had a survival of 8 months, more than the survival benefit of 3,4 months reported. ${ }^{41,42}$

\section{Conclusion}

The association of RT and Nimotuzumab in the treatment of DIPG in children and adolescents is safe and feasible and could be a therapeutic option in order to increase very long-term survival. Reirradiation is an option in case of relapse of tumor

\section{Acknowledgments}

None.

\section{Conflicts of interest}

All authors declare that there is no conflict of interest.

\section{Funding}

None.

\section{References}

1. Massimino M, Biassoni V, Miceli R, et al. Results of Nimotuzumab andVinorelbine, radiation and re-irradiation for diffuse pontine glioma in childhood. J Neurooncol. 2014;118(2):305-312.

2. Warren E. Diffuse intrinsic pontine glioma: poised for progress. Front Oncol. 2012;2:205

3. El-Khouly F, Vendhuijzen van Zanten S, Santa-Maria V, et al. Diagnostic and treatment treatment of diffuse intrinsic pontine glioma: where do we stand. J Neurooncol. 2019:145:177-184.

4. Bartels U, Wolff J, Gore L, et al. Phase II study of safety and efficacy of Nimotuzumab in pediatric patients with progressive diffuse intrinspontine glioma. Neuro-oncology. 2014;16(11):1554-1559.

5. Hoffman LM, Veldhuijzen van Zanten S, Colditz N, et al. Clinical, radiologic, pathologic and molecular characteristics of long-term survivors of diffuse intrinsic pontine glioma (DIPG). A Collaborative Report from the International and European Society for Pediatric Oncology DIPG Registries. J Clin Oncol. 2018;36(19):1963-1972.

6. Fleischhack G, Massimino M, Warmuth-Metz M, et al. Nimotuzumab and radiotherapy for treatment of newly diagnosed diffuse intrinsic pontine glioma (DIPG): a phase III Clinical Study. J Neuro-oncol. 2019;143:107-113

7. Kebudi R, Betul Cakir F, BuyuKapu B, et al. Nimotuzumab-containing regimen for pediatric diffuse pontine gliomas: a retrospective multicenter study and review of the literature. Child's Nervous System 2019;35:83-89.

8. Parsons DW, Pollack IF, Hans-Kogan D, et al. Gliomas, ependimomas and other nonembrional tumors of the Central Nervous System in Pizzo I, Philip A. Ed. Principles and Practice of Pediatric Oncology, Seventh Ed. Wolters Kluwery Hong-Kong, China. 2016;627-658.
9. Cooney T, Lane A, Bartels U, et al. Contemporary survival endpoints: an International Diffuse Intrinsic Pontine Glioma Registry study. Neuro Oncol. 2017;19(9):1279-1280.

10. Vanan MI, Eisenstat DD. DIPG in Children-what can we learn from the past. Front Oncol. 2015;5:237.

11. Carciller F. Long-term survivors of diffuse intrinsic pontine glioma (DIPG): myth or really. Trnsl Cancer Res. 2019;8(2):343-345.

12. Fried I, Hawkins C, Scheinemann K, et al. Favorable outcome with conservative treatment of children with low grade brainstem tumors. Pediatr Blood Cancer. 2012;58(4):556-560.

13. Hassan H, Pinches A, Picton SV, et al. Survival rates and prognostic predictors of high grade brainstem gliomas in childhood: a systematic review and meta-analysis. J Neuro Oncol. 2017;135(1):13-20.

14. Elmaraghi C, Bishor M, Moussa A, et al. Pediatric low grade focal brainstem glioma: outcomes of different treatment strategies and prognostic factors. Future Oncol. 2020;16(30):2401-2410.

15. Lam S, Lin Y, AuffingerB,et al. Analysis of survival in pediatric highgrade brainstem glioma: a population-based studi. J PediatrNeurosci. 2015;10(3):199-206.

16. Cohen K, Heideman R, Zhou T, et al. Temozolamide in the treatment of children with newly diagnosed diffuse intrinsic pontine gliomas: a report from the Children's Oncology Group. Neurooncol. 2011;13(4):410-416.

17. Gururamgan S, Chi S, Poussaint T, et al. Lack of efficacy of bevacizumab plus irinotecan with recurrent malignant glioma and diffuse brainstem glioma: a Pediatric Brain Tumor Consortium Study. J Clin Oncol. 2010;28(18):3069-3075.

18. Baxter P, Su J, Onar-Thomas A, et al. A Phase I/II study of Veriparib (ABT-888) with radiation and Temozolamide in newly diagnosed diffuse pontine glioma: a Pediatric Brain Tumor Consortium Study. Neurooncology. 2020;22(6):875-885.

19. Jansen M, Veldhuijezen van Zaten S, Sanchez-Aliaga E et al. Survival prediction model of children with diffuse intrinsic pontine glioma basedon clinical and radiological criteria. Neurooncology. 2015;17(1):160-166.

20. Marcus K, Dutton Sh, Barnes P, et al. A Phase I trial of etanidazole and hiperfractionated Radiotherapy in children with diffuse brainstem glioma. Int J Radiation Oncol Biol Phys. 2003;55(5):1182-1185.

21. Crotty E, Leary S, Geyer J, et al. Children with DIGP and high grade glioma treated with temozolamide, irinotecan and bevazisumab. The Seattle Chidren's Hospital Experience. J Neuro Oncol. 2020;148(3):607-617.

22. Fernandez A, Spitze K, Perez R, et al. A new monoclonal antibody for detection of EGF receptors in western blots and paraffin-embebed tissue sections. J Cell Bioch. 1992;49(2):157-165.

23. Garrido G, Tikhomitov I, Rabasa A, et al. Bivalent biding by intermediate affinity of Nimotuzumab: a contribution to explain antibody clinical profile. Cancer Biol Ther. 2011;11(4):373-382.

24. Cabanas R, Saurez G, Alert J, al. Prolonged use of Nimotuzumab in children with Central Nervous System tumors: safety and feasibility. Cancer Biother and Radiopharm. 2014;29:173-178.

25. Boland W K, Bebb G. Expert opinion, Nimotuzumab, a novel anti-EGFR monoclonal antibody that retains anti EGFR activity whileminimizing skin toxicity. Expert Opin Biol Ther. 2009;9(9):1199-1206.

26. Mateo C, Moreno C, Amour K, et al. Humanization of a mouse monoclonal antibody that blocks the epidermal growth factor receptor. Recovery of antagonist activity. Inmunotechnology. 1997;3(1);71-81.

27. Diaz-Miqueli A, Rolff J, Lemm M, et al. Radiosensitisation of U87MGbrain tumors by anti-epidermal growth factor monoclonal antibodies. Brit J Cancer. 2009;100(6):950-958 
28. Saurez-Martinez G, Bencomo-Yanes A. Nimotuzumab effective inmunotherapy for the treatment of malignant epitelial tumors. Biotecnologiaaplicada. 2014;31(2):159-167.

29. Crombet-Ramos T, Rack J, Perez R, et al. Antiproliferative, antiangiogenic and proapoptotic activity of h-R3: a humanized anti-EGFR antibody. Int J Cancer. 2002;101(6):567-575.

30. Bode U, Massimino M, Bach F, et al. Nimotuzumab treatment of malignant gliomas. Expert Opin Biol Ther. 2012;12(12):1649-1659.

31. Hargrave D, Bartels U, Bouffet E. Diffuse brainstem glioma in children: critical review of clinical trials. Lancet Oncol. 2012;7(3):241-248.

32. Alert J, Chon I, Valdes J, et al. Long term survival in diffuse infiltrative brainstem gliomas in children and adolescents treated with radiotherapy and Nimotuzumab.Int J Radiol Radiat Ther. 2018;5:267-270.

33. Alert J, Chon I, Cabanas R, et al. Radiation Therapy and Nimotuzumab in children and adolescents: a 5-years Institutional experience.Neuro Open J. 2015;2:45-50.

34. Bone U, Cabanas R, Saurez G, et al. Anti-EGFR Nimotuzumab for DIPG in recurrent or children with high grade glioma: 10 years. In Adella Ca (ED). Stem Cell Oncology, Taylor \& Francis Group, London. 2018:251-257.

35. Jackson S, Patay Z, Howarth R, et al. Clinicoradiologic characteristics of long-term survivors of diffuse intrinsic pontine glioma. $J$ Neurooncol. 2013;114(3):339-344.
36. Hersh D, Kumar R, Moore K, et al. Safety and efficacy of brainstem biopsy in children and young adults. J Neuro Surg Pediatr. 2020;1-11.

37. Gallito M, Lazarev S, Wasserman T, et al. Role of radiation therapy in the management of diffuse intrinsic pontine glioma: a systematic review. Adv Radit Oncol. 2019;4(3):520-531.

38. Hamish C, Kickingereder P, Fischer M, et al. Update on the diagnostic value and safety of stereotactic biopsy for pediatric brainstem tumors: a systematic review and meta-analysisof 735 cases. J Neurosurg. Pediatrics. 2017;20(3):261-268.

39. Jansen $M H$, van Vuurden DG, Vandertop WP, et al. Diffuse intrinsic pontine gliomas: a systematic update on clinical trials and biology. Cancer Treat Rev. 2012;38(1):27-35.

40. Tinkle Ch, Campbell K, Han Y, et al. Radiation dose response of neurological symptoms during conformal radiotherapy for diffuse intrinsic pontine glioma. J Neurooncol. 2020;147(1):195-203.

41. Tsang D, Laperriere N. Re-irradiation for pediatric tumors. Clin Oncol. 2019;31(3):191-198.

42. Janssens GO, Gandolla L, Bolle S, et al. Survival benefict for patients with diffuse intrinsic pontine glioma (DIPG) undergoing re-irradiation at first progression: a matched-cohort analysis on behalf of the SIOP-EHGG/DIPG Working Group. Eur J Cancer. 2017;73:38-47.

43. Lassaletta A, Strother D, Laperriere N, et al. Reirradiation in patients with diffuse intrinsic pontine gliomas: the Canadian experience. Pediatr Blood Cancer. 2018;65(6):e26988. 